\section{$\underset{\substack{\text { hommes } \\ \text { \& migrations }}}{ }$}

\section{Hommes \& migrations}

Revue française de référence sur les dynamiques

migratoires

$1324 \mid 2019$

Religion et discrimination

\title{
Images et dialogue citoyen
}

Retour sur le Grand Festival 2018

\section{Agnès Arquez - Roth}

\section{(2) OpenEdition \\ 1 Journals}

\section{Édition électronique}

URL : https://journals.openedition.org/hommesmigrations/8833

DOI : 10.4000/hommesmigrations.8833

ISSN : 2262-3353

\section{Éditeur}

Musée national de l'histoire de l'immigration

\section{Édition imprimée}

Date de publication : 1 janvier 2019

Pagination : 148-150

ISBN : 978-2-919040-44-5

ISSN : $1142-852 X$

\section{Référence électronique}

Agnès Arquez - Roth, «Images et dialogue citoyen », Hommes \& migrations [En ligne], 1324 | 2019, mis en ligne le 01 janvier 2019, consulté le 06 janvier 2022. URL : http://journals.openedition.org/

hommesmigrations/8833; DOI : https://doi.org/10.4000/hommesmigrations.8833 


\title{
Images et dialogue citoyen
}

\author{
Retour sur le Grand Festival 2018
}

\author{
Agnès Arquez-Roth, \\ cheffe du service Réseau \& partenariats, Établissement public du Palais de la Porte Dorée.
}

Le Musée national de l'histoire de l'immigration entretient des liens étroits avec les acteurs de la société civile depuis sa création. À l'occasion du Grand Festival en mars 2018, il a partagé avec son réseau d'acteurs des questionnements et des pratiques pour agir contre les discriminations au travers de l'image. Il a proposé de réunir ceux qui fabriquent les images, ceux qui les utilisent comme support de médiation, et ceux qui les lisent et parfois les subissent.

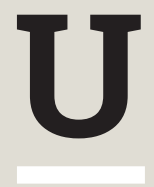

n certain nombre d'acteurs dans les collectivités territoriales et dans les associations sociales et humanitaires partagent des questionnements sur leur stratégie de communication et les difficultés à éviter les pièges des stéréotypes qui tendent, inconsciemment, à ethniciser, voire à racialiser les individus.

Plusieurs temps de rencontres ont été proposés en amont du Grand Festival afin d'éprouver le processus de fabrication des images dites d'utilité publique. L'atelier Fabrication maison a été invité à conduire des ateliers auprès de quatre structures de nature très différentes, confrontant ce processus de fabrication d'images à des publics concernés par le sujet de façon très diverse en tant que faiseur d'images ou personnes susceptibles d'être discriminées.

Fabrication maison est un collectif d'artistes qui envisage l'image comme un objet artistique porteur de significations et de questions culturelles et sociales. Il propose d'opérer un travail de pédagogie artistique pour mieux appréhender les mécanismes de l'image, ses enjeux et favoriser sa compréhension. Il implique les arts graphiques dans des projets de développements locaux, donnant à ces derniers une dimension et une lisibilité singulière, qui porte l'empreinte et la trace des personnes rencontrées.

Le protocole élaboré par leurs soins pour la déconstruction des signes et des symboles en lien avec la question du racisme et de la discrimination a très bien fonctionné quel que soit le public. Le Signe, centre national du graphisme à Chaumont, a mis à disposition une dizaine d'images créées par de grands affichistes, tels que Andrzej Klimowski, Gérard Paris Clavel, Claude Baillargeon dans le cadre de campagnes de communication des villes de Montreuil ou de Bagnolet... 
L'utilisation des images de la collection du Signe a facilité ce travail et permis aux participants de découvrir cette notion de graphisme d'utilité publique. Cette exploration de l'image a donné lieu à plusieurs phases : contextualiser/ analyser/esquisser; rechercher des mots et des images/signes adéquats ; approfondir les concepts et définir les mots racisme, discrimination et stéréotypes.

Les représentants du Centre d'hébergement d'urgence Jean Quarré (Emmaüs solidarité) ${ }^{1}$, du Centre LGBT Paris Île-de-France ${ }^{2}$, de l'association Ticket d'entrée (alphabétisation et culture) ${ }^{3}$, et du Conseil départemental de Seine-Saint-Denis (chargés de communication) 4 ont constitué, en lien avec le musée, des groupes de participants d'âge et d'horizons différents qui ont été réunis par l'atelier graphique Fabrication Maison sur leurs sites. Chaque groupe a découvert une même sélection d'images choisies par le Signe au sein de ses collections. Ils ont été conviés à éprouver le processus de fabrication d'images d'utilité publique et la complexité des signes (mot ou image) et leur utilisation.

Ensuite, les quatre groupes se sont rencontrés au Palais de la Porte Dorée, durant le Grand Festival, pour découvrir leurs différentes productions et échanger sur leurs expériences. À cette occasion, ils ont fabriqué en sérigraphie une affiche collective. Une série de cinq cartes postales issue de ces ateliers a été imprimée à l'occasion de l'événement et distribuée à l'ensemble des participants. Elles ont été mises en débat le 20 mars avec un public plus large de façon à créer de nouvelles modalités de débats. Julien Roirant, moteur de dialogue citoyen $\left(\right.$ Agoralab $\left.{ }^{5}\right)$, a orchestré la restitution publique de cette initiative à l'aide de huit ambassadeurs issus des quatre groupes du collectif Fabrication maison.

1. Le Centre d'hébergement d'urgence Jean Quarré est installé dans les locaux d'un ancien lycée du 19 e arrondissement de Paris. Confié à Emmaüs solidarité par la ville de Paris, il accueille temporairement des migrants en attente d'une solution de logement, tout en leur proposant un accompagnement en matière de formation linguistique, d'activités culturelles et sportives.

2. Le Centre LGBT de Paris et d'Île-de-France est un lieu de visibilité et d'accueil ouvert depuis 1993 dans Paris pour soutenir les personnes LGBT et leurs droits. Ouvert au public six jours sur sept, il propose de nombreux événements et accompagnements, aussi bien des événements culturels que des permanences santé et accès au droit. II est également à la tête d'un réseau de près de 80 associations.

3. Le cœur de l'activité de l'association Ticket d'entrée est de proposer des ateliers langue et culture autour de sorties culturelles à des personnes en alphabétisation. Ils interviennent dans le 19 arrondissement et plus spécifiquement dans les quartiers pris en charge par la politique de la ville. Ils développent leur action avec deux associations partenaires: Parabole et Accès à la lecture pour tous.

4. Dans le cadre de son engagement pour changer le regard sur les populations migrantes ou l'immigration, le département de Seine-Saint-Denis entend saisir l'occasion de cette manifestation pour impliquer son personnel dans la fabrication de ces images d'utilité publique et dans l'exercice à partir de celles-ci de la mise en débat. Pour cela, il réunit des chefs de projets, responsable de photothèque, de programmes sur la laïcité ou encore du pôle relations sociales, du service Via le monde et de projets sur les droits culturels.

5. Julien Roirant (Agoralab) est diplômé d'un DESS de sciences politiques (Paris I Panthéon-Sorbonne, 2000) et vice-président du Centre Primo Levi (soin aux réfugiés victimes de torture et de violence politique). II accompagne depuis 17 ans les collectivités, les institutions publiques/parapubliques et les entreprises dans la conception et la mise en œuvre de démarches participatives internes et externes. Il anime des formations, des séminaires, des tables-rondes et des débats publics. Il assure également des missions de coaching en prise de parole, en relations médias, et en gestion de crise et de conflit. 


\section{Pratique de l'image et images en pratique}

Le musée a choisi de partager ses interrogations sur les processus mis en œuvre pour contribuer à changer le regard sur les migrants, l'immigration et, plus globalement, la société française dans sa diversité. L'organisation des ateliers à destination de bénévoles et de professionnels a été confiée à Emmanuelle Roule ${ }^{6}$, graphiste/designer. Son expertise a été importante et a permis d'enrichir la réflexion avec les sémioticiens et les acteurs du Réseau. Elle a notamment créé le visuel pour la restitution publique « Image et dialogue citoyen 》.

Ces ateliers ont eu pour objectif de mettre en partage les différentes thématiques et questionnements liés à la lutte contre les discriminations et le racisme, au regard des besoins de communication et des missions d'informations développées par les collectivités territoriales et les acteurs associatifs principalement. Le principe a été le même pour les deux ateliers qui se sont tenus le 20 mars au Palais de la Porte Dorée: deux acteurs du Réseau du musée, en dialogue avec un expert en sémiotique, ont débattu et partagé leurs réflexions avec les participants.

En se plaçant du côté de celles et de ceux qui commandent des images, qui les utilisent dans le cadre de campagnes de sensibilisation et de médiations, de fortes interpellations apparaissent sur le sens donné à ces démarches. Car ces dernières réservent bien des surprises quant à la réception des images par le public ou encore le possible changement des représentations.

\section{En perspective : le Grand Festival 2019}

Dès la restitution publique du 20 mars 2018, les participants, composés à la fois d'associations, de collectivités, de migrants et d'étudiants en arts graphiques, ont exprimé le souhait de voir cette réflexion se poursuivre et d'envisager la prochaine édition du Grand Festival plus en amont. Le Grand Festival 2019 sera donc l'occasion de poursuivre cette expérimentation en s'associant cette fois-ci au Tribunal pour les générations futures, un événement imaginé par le magazine Usbek \& Rica. Cette conférence-spectacle mise en scène sous la forme d'un procès donnera la parole aux générations futures au travers un public large.

\footnotetext{
6. Emmanuelle Roule est graphiste et directrice artistique freelance depuis 2007.

En mai 2006, elle participe à l'échange de 100 jeunes artistes français en Chine, dans le cadre des croisements culturels Chine-France mis en place par le ministère des Affaires étrangères et de la Culture français. Elle a été de 2007 à 2016 membre permanent du collectif d'artistes Le parti poétique. Elle a notamment coconçu, en 2009, le projet de la Banque du miel, démarche artistique qui a essaimé dans le cadre de résidences ou d'expositions en France, en Europe et au Japon. Elle a également piloté avec Le parti poétique la programmation du lieu Zone sensible, créé par le collectif et ouvert en mai 2011 à Saint-Denis. Elle y a conçu l'événement et la direction artistique du festival intitulé Allons voir ailleurs si nous y sommes dédié à la jeune création contemporaine.

Elle est, depuis 2016, enseignante à la HEAD, Haute école d'art et de design à Genève (Suisse). En parallèle, elle développe depuis 2012, un travail d'expérimentations artistiques d'interaction entre la forme, la couleur, la lumière et la terre, notamment au travers de la pratique de la céramique. En 2017, elle fonde, avec trois autres céramistes, le collectif et atelier Gangster basé à Paris.
} 